belt fit. While 90\% reported their belt as comfortable, 21\% reported repositioning their belt. Poor lap belt fit was more likley in obese (OR2.2, 95\% CI: 1.2-4.0) and overweight drivers (OR 1.8, 95\% CI: 1.1-3.0), and females (2.2,95\% CI: 1.3-3.5). Comfort pads were associated with shorter stature (OR 1.1 95\% CI: 1.02-1.1), and cushions with belt discomfort (OR 2.5, 95\% CI: 1.1-5.6). Musculoskeletal comorbidities increased belt repositioning (OR 1.3, 95\% CI: 1.1-1.6), and comfort partially mediated this relationship $(\mathrm{p}=0.03)$. General comorbidities, increased the odds of accesory use (OR 1.2 95\% CI: 1.04-1.3).

Conclusions Older drivers face challenges achieving comfortable and correct belt fit, and many reposition belts and use comfort accesories. This may negatively impact crash protection.Older drivers need to be aware of the importance of good belt positioning, particularly those with comorbidities. The impact of accesories on injury risk needs examination.

\section{ADDRESSING ELDER ABUSE IN THE UNITED STATES: FEDERAL GUIDELINES FOR ADULT PROTECTIVE SERVICES}

${ }^{1}$ Kathy Greenlee, ${ }^{2}$ Edwin Walker, ${ }^{2}$ Stephanie Whittier-Eliason. ${ }^{1}$ U.S. Department of Health and Human Services, Administration for Community Living: ${ }^{2}$ Ibid

\subsection{6/injuryprev-2016-042156.174}

Background Elder abuse - any knowing, intentional, or negligent act by a caregiver or any other person that causes harm or a serious risk of harm to people over the age of 60 -impacts at least $6 \%$ of older adults across the globe, but only $17 \%$ of countries have collected national data to measure the scope of the problem. ${ }^{1}$ In the U.S., Adult Protective Services (APS) systems play a critical role in identifying and responding to abuse, neglect, selfneglect, and financial exploitation faced by older adults and persons with disabilities; however, APS varies across and within states, lacking uniform guidance that hinders cross-jurisdictional cooperation, information sharing, and investigation. Further, lack of standardised service provision contributes to the absence of critical supports for victims. To address this gap, in 2014, the Administration for Community Living (ACL) established the Elder Justice and Adult Protective Services Program to support state APS systems to provide consistent, evidence-based services, so victims receive quality support regardless of their state or jurisdiction.

Methods The program seeks to improve APS systems nationwide by: developing federal guidelines to provide a standard for APS services through the input of expert stakeholders; implementing a National Adult Protective Services Data Collection System; and, funding demonstration grants to 11 states to enhance their APS systems through innovative practices.

Results Preliminary results demonstrate program success, with participation from more than $75 \%$ of state and territory jurisdictions in the data collection effort, and $600+$ pages of public comments in support of the national guidelines. Phase 1 results (2 years of these collective efforts) are anticipated in June 2017.

Conclusions ACL's efforts to facilitate the development of a coordinated, national approach to APS systems shows promise for enhancing state and local responses to investigating and responding to abuse, neglect, and exploitation of older adults, incorporating data collection to guide current practice and future research, evidence-based practices, and uniform response standards.

\section{NOTE}

1 Global Status Report on Violence Prevention 2014. World Health Organisation, 2014.

\section{INJURIOUS FALLS AND SUBSEQUENT ADVERSE DRUG EVENTS AMONG ELDERLY - A SWEDISH POPULATION- BASED MATCHED CASE-CONTROL STUDY}

${ }^{1,2}$ Christian Rausch, 'Lucie Laflamme, ${ }^{2}$ Sophia de Rooij, 'Jette Möller. 'Karolinska Institutet Sweden; ${ }^{2}$ University Medical Centre Groningen, The Netherlands

\subsection{6/injuryprev-2016-042156.175}

Background Various factors, including chronic diseases, multiple morbidity and polypharmacy, but also injuries like falls can put elderly at increased risks for adverse drug events (ADE). Despite numerous studies underlining the significance of healthy ageing, epidemiologic studies on community dwelling populations remain scarce in this field. In our study we determine the subsequent association between injurious falls and ADEs among older people, while recognising the role of number and type of medications, as well as co-morbidity.

Methods We used a matched case-control design for our study among Swedish residents 60 years and older. ADEs by unintentional poisoning resulting in hospitalisation or death were considered as cases. These were extracted from the National Patient Register (NPR) and the Cause of Death Register from January 2006 to December 2009. Cases were matched with four controls by sex, age and residential area. The medication prescribed during the four month period prior to index date, was extracted from the Swedish Prescribed Drug Register (SPDR), while previous episodes of injurious falls were extracted from the NPR. For our analysis we used conditional logistics regression and estimated our effects with odds rations (OR) and 95\% confidence intervals (CI). In the analysis, we adjusted for confounders, including comorbidity.

Results Within six-month after an injurious fall, we found a three-fold increased risk for an ADE among older people. Although the risk for an ADE after a fall injury is significantly high over the whole period, it was highest in the one to three weeks period immediately after an injurious fall. Younger older individuals (60-79 year), were at a higher risk than older ones $(80+$ year). Older people with an ADE up to three weeks following a fall injury had a tendency to have been prescribed a higher number of medications, but were less likely to have a previous medical history involving other fall-related hospitalizations.

Conclusions Older people with an injurious fall are at an increased risk for a subsequent ADE. The period after an injurious fall could serve as potential point for the prevention of ADEs. Falls are common among the older population group, and the medicinal options for treatments after a fall should be carefully regarded by physicians and their patients in consideration of the potential risk for ADEs. 


\section{Child and Adolescent Safety}

Parallel Mon 3.2

\section{TEACHABLE MOMENTS}

${ }^{1}$ Peter Spitzer, ${ }^{1,2}$ Holger Till. ${ }^{1}$ Center for Childhood Accident Research and Injury Prevention, Graz, Austria; ${ }^{2}$ Medical University of Graz, Department of Ped. Surgery, Austria

\subsection{6/injuryprev-2016-042156.176}

Background Every year more than 12,000 children aged 0 to 14 visit the Graz Department of Paediatrics Emergency Room after an accident. Additionally, more than 5,000 babies are delivered at the Department of Obstetrics - both departments are located on the State Hospital's campus in Graz.

Objectives How can we reach parents of young children with safety messages? When is the best time to teach parents about child safety?

Results From our long-term experience we look to very important teachable moments to inform and teach parents of young children about child safety.

The first moment is around birth. For this purpose, we adopted the idea of a child safety house from Australia and built the first Austrian Child Safety House next to the Department of Paediatrics in Graz. The house makes it possible to show expectant and new parents how to make their homes safe.

The second moment is when parents accompany their children after an accident as inpatients in the hospital. And for that purpose we adopted a best practice project from Israel, called bedside counselling. This project aims first to inquire after the exact circumstances of the accident; second to improve parents' level of knowledge about safety through a personal discussion with a safety expert and with information sheets that cover limited age ranges; and third to monitor the effectiveness of the counselling with a follow-up phone call. This project was very effective for parents of children up to the age of 8 . Moreover, we discovered that after an accident, even if the injury was not severe, parents were more traumatised than we had expected.

Conclusions The bedside counselling program and the child safety house tours demonstrated that these critical moments of parents' lives should be used to make their homes safer for babies and young children and to motivate them to think about safety more broadly.

\section{HOW TO CHOOSE WHO TO FOCUS ON? NATIONAL STUDY TO IDENTIFY RISK GROUPS FOR CHILD INJURY}

Oshri Weiss, Avichai Baram, Natalie Nir, Shira Kislev, Orly Silbinger. Beterem-Safe Kids Israel

\subsection{6/injuryprev-2016-042156.177}

Background Identifying populations at high risk for injury is a key step in developing effective strategies to reduce overall burden of injuries and gaps in injury rates between different segments of the population. This need is even more pressing in times of economic crises. Israel's population is diverse representing different ethnic, socio-economic, religious and cultural groups. Comparing child injury risks among different segments of the Israeli population enables us to identify high risk groups and their proportion of injury burden.

Methods Child population in Israel was divided into 27 groups based on cultural and ethnic affiliation (Jews, Arabs, Haredi, and Bedouin), socio-economic status (SES), age group and region based on municipal level data. We calculated the rate of child injury per group using mortality and hospitalisation data. The relative proportion of each group's injury burden to maximise the impact of future targeted interventions was calculated. Finally, we used cluster analysis to rank each group into one of four injury risk groups.

Results Bedouin children of low SES aged 0-4 years, in the southern region, were identified as top priority for intervention (mortality rate of 29.3 per 100,000 , hospitalisation rate 807.8 days per 10,000$)$. This group is $1.0 \%$ of the child population yet carries $8.3 \%$ of the mortality burden. The lowest priority cluster, constituting $85 \%$ of the child population includes the entire Jewish population at all SES levels as well as a minority of the Arab children (mortality rate of 2.5 per 100,000 ).

Conclusions This study points at young Bedouin children as the highest risk group for injuries in Israel. Based on these findings they have been targeted for prevention efforts by national and local authorities for 2016-2020. Focusing on feasible, cost-effective, proven, and tailored interventions for at risk populations, in cooperation with community leaders, may achieve considerable and lasting improvement in safety.

\section{INJURY PREVENTION IN FINLAND AMONG PEOPLE UNDER 25 YEARS 2009-2014}

Ulla Korpilahti, Laura Kolehmainen, Satu Pajala, Anne Lounamaa. 4 National Institute for Health and Welfare, Finland

\subsection{6/injuryprev-2016-042156.178}

Background National action plan for injury prevention among children and youth in Finland was launched in 2009. National Institute for Health and Welfare (THL), national research and expert agency under the Ministry of Social Affairs and Health (MSAH), has coordinated the program. National action plan includes 216 objectives and proposed measures to promote and prevent accidental injuries and suicides.

Description of the problem Around 122 Finnish children and young people under the age of 25 die annually in accidental injuries and 13,500 are hospitalised (2011-2013). Accidental injuries remain the leading cause of death under the age of 25 . Majority of the deaths (83\%) occur to 15-24 year olds. Around 25 percent of all 15-24 year-olds who die from accidental injury are intoxicated. Traffic accidents are the most common accidental cause of death in this age group. Accidental falls are causing most of the treatment inpatients periods in hospital, respectively.

Results Despite the continuous decrease of deaths from accidental injuries since the 1970's, accidental injuries causes major health losses among children and youth. The objectives, measures and their implementation and responsibilities of the National action plan are divided into a number of different experts and government departments. Legislation and national policy guidelines, as well as statistics and databases are generally at a good level in Finland. The monitoring of the management and implementation should be paid more attention.

Conclusions In order to be successful a National action plan requires high level recognition and adequate resources together with systematic coordination. National action plan is essential to be approved by the appropriate ministries and politicians. That made possible and empowered the national level activities and promoted a network of cooperation between the different actors. There is need for targeted accidental injury prevention especially for youth from 15 to 25 years. 